\title{
Transport in charged colloids driven by thermoelectricity
}

\author{
Alois Würger \\ CPMOH, Université Bordeaux $1 \&$ CNRS, 351 cours de la Libération, 33405 Talence, France
}

\begin{abstract}
We study the thermal diffusion coefficient $D_{T}$ of a charged colloid in a temperature gradient, and find that it is to a large extent determined by the thermoelectric response of the electrolyte solution. The thermally induced salinity gradient leads in general to a strong increase with temperature. The difference of the heat of transport of co-ions and counterions gives rise to a thermoelectric field that drives the colloid to the cold or to the warm, depending on the sign of its charge. Our results provide an explanation for recent experimental findings on thermophoresis in colloidal suspensions.
\end{abstract}

PACS numbers: 66.10.C, 82.70.-y,47.57.J-

Introduction. Colloidal suspensions in a non-uniform electrolyte show a rich and surprising transport behavior. Upon applying an electric field or a chemical or thermal gradient on a macromolecular dispersion, one observes migration of its components and a non-uniform distribution in the stationary state. The physical mechanisms of electrophoresis and diffusiophoresis are well understood [1, 2] and widely used in biotechnology and microfluidic applications [3, 4].

The situation is less clear concerning transport driven by a thermal gradient. There is no complete description for the underlying physical forces, and even the sign of the thermophoretic mobility lacks a rationale so far. It had been known for a while that in some colloidal suspensions the particles move to the cold, and in others to the warm, corresponding to a positive and negative Soret effect, respectively [5 7. Recent experiments on aqueous solutions of lysozyme protein [8, 9], polystyrene beads [9 12, micelles [11, DNA [13], and Ludox particles [14] revealed a surprisingly similar temperature dependence in the range $T=0 \ldots 80^{\circ} \mathrm{C}$. In all cases, an inverse Soret effect occurs at low $T$, changes sign at some intermediate value $T^{*}$, and seems to saturate above $50{ }^{\circ} \mathrm{C}$. On the other hand, a large negative thermophoretic mobility has been reported for charged latex spheres in a buffered solution at weak acidity and low salinity [10; adding $\mathrm{LiCl}$ or $\mathrm{NaCl}$ results in a change of sign and a transport velocity that depends significantly on the cation. These features strongly suggest a single mechanism related to the electric properties of the colloid; the relevance of the thermoelectric effect for colloidal suspensions has been pointed out recently [10].

A thermal gradient modifies the solute-solvent interactions and drives the particle at a velocity 15

$$
\mathbf{u}=-D_{T} \boldsymbol{\nabla} T,
$$

the coefficient $D_{T}$ being of the order of $\mu \mathrm{m}^{2} / \mathrm{Ks}$. Like any linear transport coefficient in a viscous fluid, the thermophoretic mobility $D_{T}$ has to be evaluated by equilibrating the forces exerted by the particle on the surrounding fluid with the dissipative stress; the hydrodynamic treatement is well known, in terms of Stokes' equation with boundary layer approximation [16 21].

The present paper deals with a non-uniform electrolyte solution. We start by showing how the Soret effect of the mobile ions leads to a salinity gradient and a macroscopic thermoelectric field [22, 23]. Then we add charged colloidal particles and study how their thermal diffusion coefficient $D_{T}$ depends on the electrolyte Soret and thermoelectric effects.

Consider an electrolyte with monovalent ions of charge $q_{i}=z_{i} e$ and densities $n_{i}$. The current of each species,

$$
\mathbf{J}_{i}=-D_{i}\left(\nabla n_{i}+n_{i} \frac{Q_{i}^{*}}{k_{B} T^{2}} \nabla T-n_{i} \frac{q_{i} \mathbf{E}_{\infty}}{k_{B} T}\right),
$$

comprises normal diffusion with the Einstein coefficient $D_{i}$, thermal diffusion with the ionic heat of transport $Q_{i}^{*}$, and an electric-field term. In the stationary state $\mathbf{J}_{i}=0$, one observes a gradient of the overall electrolyte strength $n_{0}=\frac{1}{2} \sum_{i} n_{i}$ and a thermoelectric field $\mathbf{E}_{\infty}$. Both are well-defined macroscopic quantities, whereas the corresponding charge separation $\rho_{\infty}=\sum_{i} q_{i} n_{i}$ varies with the inverse system size and thus is negligible [22, 23. With $\sum_{i} \mathbf{J}_{i}=0$ and $\rho_{\infty} \rightarrow 0$ one readily obtains the salinity gradient

$$
\frac{\nabla n_{0}}{n_{0}}=-\alpha \frac{\nabla T}{T},
$$

where the reduced Soret coefficient $\alpha$ of the electrolyte solution is given by the mean heat of transport

$$
\alpha=\sum_{i} \alpha_{i} \frac{n_{i}}{n_{0}}, \quad \alpha_{i}=\frac{Q_{i}^{*}}{2 k_{B} T}
$$

The thermoelectric field is calculated from the condition of zero electrical current $\sum_{i} q_{i} \mathbf{J}_{i}=0$; taking $\rho_{\infty} \rightarrow 0$ one finds 23 .

$$
e \mathbf{E}_{\infty}=\delta \alpha k_{B} \nabla T
$$

with the dimensionless coefficient

$$
\delta \alpha=\sum_{i} z_{i} \alpha_{i} \frac{n_{i}}{n_{0}} .
$$

These relations are readily generalzed to higher valencies; for a binary electrolyte they reduce to $\alpha=\alpha_{+}+\alpha_{-}$and $\delta \alpha=\alpha_{+}-\alpha_{-}$. The origin of the field $\mathbf{E}_{\infty}$ is similar to thermoelectricity in metals, where the Seebeck coefficient 
is defined as the ratio of induced voltage $\Delta \psi_{\infty}$ and temperature difference; with typical values $Q_{i}^{*} \sim \mathrm{kJ} / \mathrm{Mol}[24$ ] one finds $\Delta \psi_{\infty} / \Delta T \sim 100 \mu \mathrm{V} / \mathrm{K}$. In dilute electrolyte solutions, the ionic heat of transport $Q^{*}$ arises from specific hydration effects [22]; at salt concentrations beyond a few $\mathrm{mMol} / \mathrm{l}$ electrostatic interactions become important and result in intricate dependencies on temperature and salinity [25]27].

Force density. Now we consider a suspended colloidal particle of radius $a$ and surface charge density $e \sigma$. Because of the applied thermal gradient, the permittivity $\varepsilon$ and the Debye length $\lambda$ vary along the particle surface, and so do the electric potential $\psi$, the field $\mathbf{E}=-\nabla \psi$, and the ion densities in the boundary layer. The electric forces lead to a relative velocity $\mathbf{v}$ of the charged fluid in the vicinity of the particle, with additional ion currents $\delta \mathbf{J}_{i}=\delta n_{i} \mathbf{v}-D_{i}\left(\boldsymbol{\nabla} \delta n_{i}-\delta n_{i} q_{i} \mathbf{E} / k_{B} T\right)$. Typical velocities $v \sim \mu \mathrm{m} / \mathrm{s}$ correspond to very small Peclet numbers $\mathrm{Pe}=v a / D_{i} \ll 1$; thus the convection term $\delta n_{i} \mathbf{v}$ may be neglected, and the excess ion densities $\delta n_{i}$ in the boundary layer are given by Poisson-Boltzmann theory, $\delta n_{i}=n_{i}\left(e^{-q_{i} \psi / k_{B} T}-1\right)$, where $n_{i}$ describe the pure electrolyte discussed above. The local charge and excess ion densities read $\rho=-2 e n_{0} \sinh \hat{\psi}$ and $n=2 n_{0}(\cosh \hat{\psi}-1)$, with $\hat{\psi}=e \psi / k_{B} T$.

Thus calculating the thermophoretic mobility reduces to the hydrodynamics in the charged double layer 2 . The fluid motion is described by Stokes' equation $\eta \nabla^{2} \mathbf{v}=$ $\boldsymbol{\nabla}\left(P_{0}+n k_{B} T\right)-\mathbf{f}_{0}$, where $\eta$ is the solvent viscosity and $P_{0}$ its pressure. The force density $\mathbf{f}_{0}=\rho\left(\mathbf{E}+\tilde{\mathbf{E}}_{\infty}\right)-\frac{1}{2} E^{2} \boldsymbol{\nabla} \varepsilon$ consists of a charge term with local and macroscopic electric fields, and a dielectric term 28. (Typical values $E \sim 10^{7} \mathrm{~V} / \mathrm{m}$ and $E_{\infty} \sim 10^{2} \mathrm{~V} / \mathrm{m}$ imply $E_{\infty} \ll E$.) Rewriting Stokes' equation as $\eta \boldsymbol{\nabla}^{2} \mathbf{v}=\boldsymbol{\nabla} P_{0}-\mathbf{f}$ and spelling out the gradients in $\mathbf{f}=\mathbf{f}_{0}-\boldsymbol{\nabla}\left(n k_{B} T\right)$, one finds

$$
\begin{aligned}
\mathbf{f}= & -\left(\rho \psi+n k_{B} T\right) \frac{\boldsymbol{\nabla} T}{T} \\
& -\frac{E^{2}}{2} \nabla \varepsilon+n k_{B} T \frac{\nabla n_{0}}{n_{0}}+\rho \tilde{\mathbf{E}}_{\infty} .
\end{aligned}
$$

Note that the force density arises from the slowly varying macroscopic solvent parameters $T, \varepsilon, n_{0}$, and the thermoelectric field $\tilde{\mathbf{E}}_{\infty}$. The permittivity of water being much larger then that of the particle, $\varepsilon \gg \varepsilon_{P}$, it modifies $\tilde{\mathbf{E}}_{\infty}$ close to the interface and, in particular, enhances the parallel component $\tilde{E}_{\infty}=\frac{3}{2} E_{\infty}$.

Following standard arguments [2], we solve Stokes' equation in boundary-layer approximation, that is, for particles larger than the Debye length $\lambda \ll a$. With local coordinates $x$ and $z$ parallel and perpendicular to the surface, the force balance in normal direction reads $\partial_{z} P_{0}-f_{z}=0$. The normal force vanishes, $f_{z}=0$, implying constant $P_{0}$. Integrating the equation for the parallel component $\eta \partial_{z}^{2} v_{x}+f_{x}=0$ with Stokes boundary conditions, one finds the fluid velocity well beyond the charged layer,

$$
v_{B}=\frac{1}{\eta} \int_{0}^{\infty} d z z f_{x} .
$$

In the laboratory frame, the fluid is immobile at infinity, and the particle moves in the opposite direction with the average boundary velocity, $\mathbf{u}=-\left\langle\mathbf{e}_{x} v_{B}\right\rangle[2$.

All forces in $(7)$ are proportional to the parallel component $T_{x}=\partial_{x} T$ of the thermal gradient. Inserting the reduced Soret and Seebeck coefficients $\alpha$ and $\delta \alpha$ and rewriting $\partial_{x} \varepsilon$ in terms of the logarithmic derivative $\tau=-d \ln \varepsilon / d \ln T$, we obtain

$$
f_{x}=\left(\frac{\tau \varepsilon E^{2}}{2 k_{B} T}-\frac{\rho \psi}{k_{B} T}+(\alpha-1) n+\frac{3}{2} \delta \alpha \frac{\rho}{e}\right) k_{B} T_{x} .
$$

The contribution in $\alpha$ accounts for the variation of the salinity $n_{0}$ along the thermal gradient. The term proportional to $\delta \alpha$ describes the effect of the electric field $E_{\infty}$; it depends on the sign of the screening cloud and thus of the particle's charge $\sigma$. Since all contributions in (9) are of similar magnitude, the force $f_{x}$ and thus the transport coefficient $D_{T}$ may take both signs, depending on the particle valency and the electrolyte properties. With the heat of transport $Q^{*}$ measured for ions in electrolyte solutions, both $\alpha$ and $\delta \alpha$ take values of the order unity that may be positive or negative. For the case $\alpha=0=\delta \alpha$, as assumed implicitly in [16 20, $f_{x}$ is strictly positive and leads to thermophoretic motion opposite to the thermal gradient, $D_{T}>0$.

Thermally driven transport. We have not yet specified the electric potential. Gouy-Chapman theory for (almost) flat surfaces gives $\hat{\psi}=4 \operatorname{artanh}\left(\nu e^{-z / \lambda}\right)$ [29], where the properties of the charged particle-fluid interface are condensed in the number $\nu=\left(1+1 / \hat{\sigma}^{2}\right)^{\frac{1}{2}}-1 / \hat{\sigma}$; the dimensionless coupling parameter

$$
\hat{\sigma}=2 \pi \sigma \lambda \ell_{B}
$$

depends on the charge density $\sigma$, the Debye length $\lambda=$ $\left(8 \pi n_{0} \ell_{B}\right)^{-\frac{1}{2}}$, and the Bjerrum length $\ell_{B}=e^{2} /\left(4 \pi \varepsilon k_{B} T\right)$. In the weak-charge limit one readily recovers the potential in Debye-Hückel approximation $\psi=(\lambda \sigma e / \varepsilon) e^{-z / \lambda}$.

With the explicit expressions for the potential $\psi$, the electric field $E=-\partial_{z} \psi$, and the charge and ion densities $\rho$ and $n$, the integral in (8) can be performed analytically,

$$
v_{B}=\frac{1}{\eta} \frac{k_{B} T_{x}}{8 \pi \ell_{B}} \hat{C},
$$

where the dimensionless quantity

$$
\hat{C}=\hat{\zeta}^{2}+8(\alpha+\tau-3) \ln \cosh \frac{\hat{\zeta}}{4}-3 \delta \alpha \hat{\zeta}
$$

is given as a function of the reduced surface potential $\hat{\zeta}=\hat{\psi}(0)$. The relation to the coupling parameter $\hat{\sigma}$ is established by

$$
\hat{\zeta}=2 \operatorname{arsinh} \hat{\sigma} \text {. }
$$




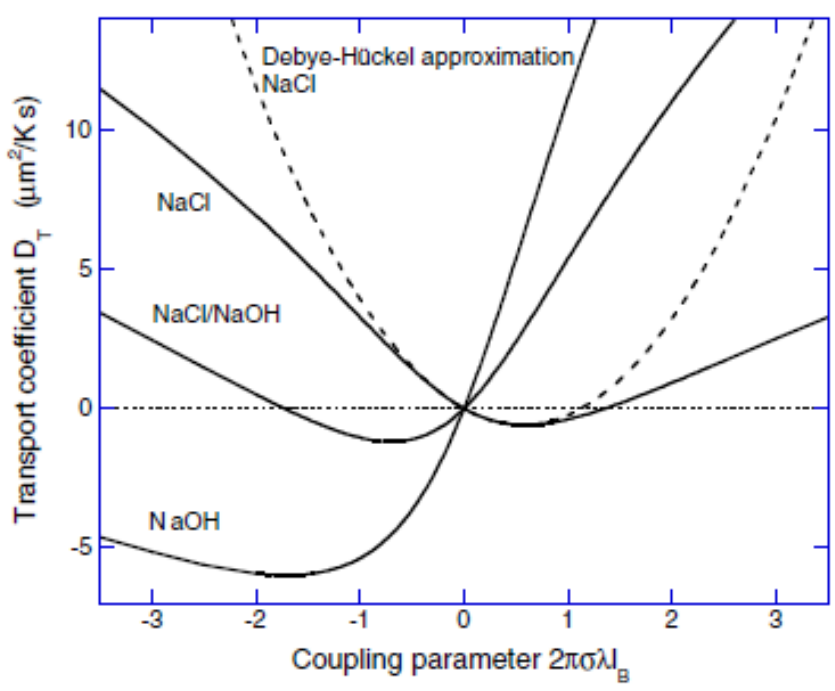

FIG. 1: Transport coefficient $D_{T}$ for different electrolytes as a function of the reduced coupling parameter $\hat{\sigma}=2 \pi \sigma \lambda \ell_{B}$. The full and dashed lines give Eqs. (13) and 14, respectively. With the numbers of Ref. [24] and Eqs. (4) and (6) one has $\alpha=0.8, \delta \alpha=0.6$ for NaCl; $\alpha=2.45, \delta \alpha=-1.05$ for equimolar $\mathrm{NaCl} / \mathrm{NaOH}$ solution; $\alpha=4.1, \delta \alpha=-2.7$ for $\mathrm{NaOH}$.

The most relevant experimental control parameters are the Debye length and the surface charge density.

The transport coefficient $D_{T}$ is obtained by averaging $v_{B}$ over the orientation of the surface with respect to the applied thermal gradient, $\mathbf{u}=-\left\langle v_{B} \mathbf{e}_{x}\right\rangle$ [2]. With $\left\langle T_{x} \mathbf{e}_{x}\right\rangle=\frac{2}{3} \boldsymbol{\nabla} T$ and including the factor $\xi=3 \kappa_{S} /\left(2 \kappa_{S}+\kappa_{P}\right)$ accounting for the thermal conductivity ratio of solvent and particle [19, one finds

$$
D_{T}=\xi \frac{k_{B}}{12 \pi \eta \ell_{B}} \hat{C}
$$

Eq. 13 constitutes the main result of this paper and provides the explicit dependence on the electric properties of solute and solvent, in terms of the surface charge density $\sigma$, the permittivity $\varepsilon$, the Debye length $\lambda$, and the electrolyte Soret and Seebeck coefficients $\alpha$ and $\delta \alpha$.

In the case of weak-coupling, $|\hat{\sigma}| \ll 1$, we use $\hat{\zeta}=$ $2 \hat{\sigma}$, expand 12 to quadratic order, $\hat{C}=\hat{\sigma}^{2}(1+\alpha+$ $\tau)-6 \hat{\sigma} \delta \alpha$, and obtain the transport coefficient in Debye-

TABLE I: Heat of transport $Q_{i}^{*}$ and reduced Soret coefficient $\alpha_{i}$ at room temperature for dilute systems. The values $Q_{i}^{*}$ are taken from Ref. 24. The parameters $\alpha_{i}$ follow from Eq. (4).

\begin{tabular}{|l|c|c|c|c|c|c|}
\hline Ion & $\mathrm{H}^{+}$ & $\mathrm{Li}^{+}$ & $\mathrm{K}^{+}$ & $\mathrm{Na}^{+}$ & $\mathrm{OH}^{-}$ & $\mathrm{Cl}^{-}$ \\
\hline$Q_{i}^{*}(\mathrm{~kJ} / \mathrm{Mol})$ & 13.3 & 0.53 & 2.59 & 3.46 & 17.2 & 0.53 \\
\hline$\alpha_{i}$ & 2.7 & 0.1 & 0.5 & 0.7 & 3.4 & 0.1 \\
\hline
\end{tabular}

TABLE II: Coupling parameter $\hat{\sigma}=2 \pi \sigma \lambda \ell_{B}$ calculated from the experimental parameters of several systems.

\begin{tabular}{|l|c|c|c|c|}
\hline & $\lambda / \mathrm{nm}$ & $\sigma / \mathrm{nm}^{-2}$ & $\zeta / \mathrm{mV}$ & $\hat{\sigma}$ \\
\hline Protein T4L [9] & & & $26 \ldots 67$ & $0.5 \ldots 1.8$ \\
\hline PS beads [9] & & & $-85 \ldots 96$ & -3 \\
\hline SDS micelles [6] & $0.5 \ldots 2.5$ & $\sim-0.2$ & & $-0.6 \ldots 3.1$ \\
\hline Ludox particles [14] & $0.5 \ldots 8$ & -0.04 & & $-0.1 \ldots 1.4$ \\
\hline
\end{tabular}

Hückel approximation,

$$
D_{T}=\frac{\xi e^{2}}{12 \eta \varepsilon T}\left((1+\alpha+\tau) \sigma^{2} \lambda^{2}-\delta \alpha \frac{3 \sigma \lambda}{\pi \ell_{B}}\right)
$$

The first term proportional to $1+\alpha+\tau$ agrees with that obtained previously in 21. For $\alpha=0=\delta \alpha$ and in the limit $\lambda / a \rightarrow 0$, our Eq. 13 agrees with the result of [19, and Eq. 14 confirms the law $D_{T} \propto \lambda^{2}$ obtained in Refs. 6, 16 18. A linear variation occurs for small particles [21, 30 32], i.e., in the limit opposite to that treated here.

Discussion. The main result of the present work concerns the effect of the thermoelectric field. In the absence of electrolyte Soret and Seebeck effects $(\alpha=0=\delta \alpha)$, the coefficient $D_{T}$ is strictly positive, i.e., a temperature gradient drives the suspended particles towards colder regions. An inverse effect $\left(D_{T}<0\right)$ occurs for a sufficiently negative Soret coefficient $\alpha$, or if the product $\delta \alpha \hat{\zeta}$ takes a positive value. In physical terms, $\alpha<0$ means a higher salinity in warmer regions of the solution, whereas $\delta \alpha$ describes the direction and magnitude of the thermoelectric field with respect to the thermal gradient.

The numbers of Table I and Ref. 24] suggest that protons are the main source of the thermoelectric effect. The crucial role of the electrolyte composition is confirmed by the experimental observation that $\mathrm{pH}$ and the presence of protonated buffers significantly influence thermophoresis [8 14. This is illustrated in Fig. 1 for $\mathrm{NaCl} / \mathrm{NaOH}$ solution with different content of the strong base sodium hydroxide. Sodium chloride has positive Soret and Seebeck coefficients, thus a slightly negative $D_{T}$ occurs for positively charged colloids. The large Soret strength of $\mathrm{OH}^{-}$results in $\delta \alpha<0$; then the thermoelectric field $\mathbf{E}_{\infty}$ is opposite to the thermal gradient and drives a negatively charged colloidal particle to higher $T\left(D_{T}<0\right)$.

For $\mathrm{NaCl}$ solution we compare the Gouy-Chapman or strong-coupling expression (13) and the Debye-Hückel approximation (14); according to the curves in Fig. 1, the latter works well for $|\hat{\sigma}|<\frac{1}{2}$, but ceases to be valid at $|\hat{\sigma}| \sim 1$. Most experimental systems carry rather high charge and surface potential $\zeta=\hat{\zeta} k_{B} T / e$, thus requiring a strong-coupling description; the numbers for $\hat{\sigma}$ in Table II imply that Debye-Hückel approximation fails for these systems.

Fig. 2 illustrates the effect of the electrolyte composition at low acidity as a function of the added amount of $\mathrm{NaCl}$ or $\mathrm{LiCl}$. The points present experimental data 


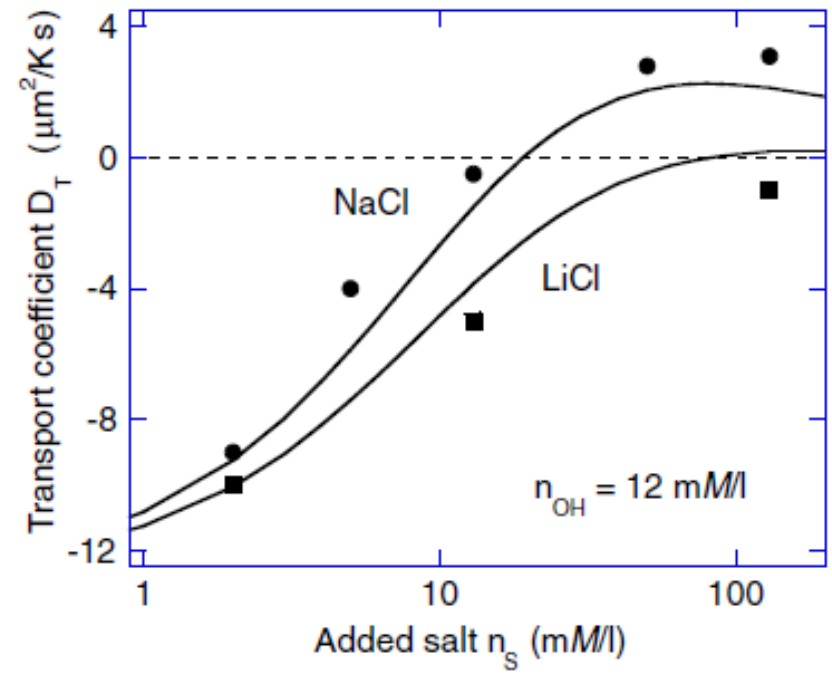

FIG. 2: Thermophoretic mobility at large pH.as a function of added salt concentration $(\mathrm{NaCl}$ or $\mathrm{LiCl})$. The data points are taken from Fig. 5a and 5b of Ref. [10]; they are obtained for polystyrene beads of radius $a=13 \mathrm{~nm}$ in a CAPS buffered electrolyte solution at fixed $\mathrm{pH}$. The curves are calculated from Eq. 13 with the ionic Soret coefficients of Table 1 for $\mathrm{Na}$ or $\mathrm{Li}, \mathrm{Cl}$, and $\mathrm{OH}$, assuming a constant charge density $\sigma=-0.12 \mathrm{~nm}^{-2}$. The hydroxide concentration $n_{\mathrm{OH}}=12$ $\mathrm{mMol} / \mathrm{l}$ corresponds to $\mathrm{pH}=10.3$. from Ref. [10] for 26-nm polystyrene beads in a CAPS buffered electrolyte solution. Since the Soret parameters for the buffer molecules are not known, only $\mathrm{Na}, \mathrm{Li}, \mathrm{Cl}$, $\mathrm{OH}$ are taken into account, with the values of table 1 . At low salinity the thermophoretic mobility is to a large extent determined by the thermoelectric field of hydroxide ions and takes a large negative value. Adding salt weakens this effect through the decreasing relative weight of $\alpha_{\mathrm{OH}}$ in the coefficients $\alpha$ and $\delta \alpha$. For $n_{S} \gg n_{\mathrm{OH}}$, the $\mathrm{pH}$ value becomes irrelevant for the thermophoretic mobility.

We conclude with a discussion of the temperature dependence of $D_{T}$. The Soret coefficients of the alkali chloride serie show a slope $d \alpha / d T=0.03 \mathrm{~K}^{-1}$ [25 27]. Assuming the same law to hold for $\delta \alpha(T)$ and using the values of Table 1 at $25{ }^{\circ} \mathrm{C}$, we obtain a good fit for the data of 11 on polystyrene beads in a $4 \mathrm{mMol} / \mathrm{l} \mathrm{NaCl}$ solution in the range from 0 to $40^{\circ} \mathrm{C}$, and in particular, the change of sign of $D_{T}$ at $T=5{ }^{\circ} \mathrm{C}$. For comparison, the Debye length $\lambda \sim \sqrt{T \varepsilon}$ and the permittivity $d \tau / d T<0.01 \mathrm{~K}^{-1}$ depend weakly on $T$; the viscosity $d \ln \eta / d T \sim-0.02 \mathrm{~K}^{-1}$ [34] provides an overall factor to $D_{T}$ but does not affect its sign. The temperature variation of the ionic Soret coefficients is strongly correlated with the thermal expansivity $\beta$ of the solvent [25]. In addition to the electrostatic term, the van der Waals interaction could contribute to $D_{T}$ a term proportional to $\beta[11,33$, with a temperature dependence similar to that of $\alpha$.
[1] S.S. Dukhin, B.V. Derjaguin, in: E. Matijevic (Ed.) Surface and Colloid Science Vol 7, Wiley New York, (1974)

[2] J. L. Anderson, Ann. Rev. Fluid Mech. 21, 61 (1989)

[3] J.L. Viovy, Rev. Mod. Phys. 72, 813 (2000)

[4] H.A. Stone et al., Ann. Rev. Fluid Mech. 36, 381 (2004)

[5] W. Köhler, S. Wiegand (eds.): Thermal Nonequilibrium Phenomena in Fluid Mixtures, Springer (2001)

[6] R. Piazza, A. Guarino, Phys. Rev. Lett. 88, 208302 (2002)

[7] G. Demouchy et al., J. Phys. D: Appl. Phys. 37, 1417 (2004)

[8] S. Iacopini, R. Piazza, Europhys. Lett. 63, 247 (2003)

[9] S.A. Putnam et al., Langmuir 23, 9221 (2007)

[10] S.A. Putnam, D.G. Cahill., Langmuir 21, 5317 (2005)

[11] S. Iacopini et al., EPJ E 19, 59 (2006)

[12] M. Braibanti et al., Phys. Rev. Lett. 100, 108303 (2007)

[13] S. Duhr, D. Braun, PNAS 103, 19678 (2006)

[14] H. Ning et al., Langmuir 24, 2426 (2008)

[15] S.R. de Groot, P. Mazur, Non-equlibrium Thermodynamics, North Holland Publishing, Amsterdam (1962)

[16] E. Ruckenstein, J. Colloid Interface Sci. 83, 77 (1981)

[17] A. Parola, R. Piazza, EPJ E 15, 255 (2004)
[18] S. Fayolle et al., Phys. Rev. E 77 (2008)

[19] K.I. Morozov, JETP 88, 944 (1999)

[20] A. Würger, Phys. Rev. Lett. 98, 138301 (2007)

[21] S.N. Rasuli, R. Golestanian, arXiv:0708.0090v1 (2007)

[22] E.D. Eastman, J. Am. Chem. Soc. 50, 283 and 292 (1928)

[23] G. Guthrie et al., J. Chem. Phys. 17, 310 (1949)

[24] J.N. Agar et al., J. Phys. Chem. 93, 2082 (1989)

[25] D.R. Caldwell, J. Phys. Chem. 77, 2004 (1973)

[26] D.R. Caldwell, S.A. Eide, Deep Sea Res. 28A, 1605 (1981)

[27] F.S. Gaeta et al., J. Phys. Chem. 26, 2967 (1982)

[28] L. D. Landau, E. M. Lifshitz, Electrodynamics of Continuous Media, Elsevier (1987)

[29] P.C. Hiemenz, R. Rajagopalan, Principles of Colloid and Surface Chemistry, Dekker (1997)

[30] E. Bringuier, A. Bourdon, PRE 67, 011404 (2003)

[31] S. Fayolle et al., Phys. Rev. Lett. 95, 208301 (2005)

[32] J.K.G. Dhont et al., Langmuir 23, 1674 (2007)

[33] H. Brenner, Phys. Rev. E 74, 036306 (2006)

[34] CRC Handbook of Chemistry and Physics, 55th edition, CRC Press (1974) 